\title{
Indeterminate results of QuantiFERON TB-2G test performed in routine clinical practice
}

\author{
Y. Kobashi, T. Sugiu, K. Mouri, Y. Obase, N. Miyashita and M. Oka
}

ABSTRACT: The present authors assessed risk factors that can promote indeterminate results of QuantiFERON TB-2G (QFT-2G; Cellestis Ltd, Carnegie, Australia) tests.

The subjects were 704 patients with suspected tuberculosis (TB) and latent TB infection between January 2005 and December 2007. The QFT-2G test and the tuberculin skin test (TST) were performed for all subjects. If the results of the QFT-2G test were indeterminate, the test was repeated within 1 month.

In total, 72 (10.2\%) patients showed indeterminate results on the QFT-2G test. Indeterminate results were due to positive control failure in 68 (88.9\%) patients and negative control failure in four patients. The results of the TST were negative for 64 patients showing indeterminate results, the remaining eight patients showed a positive response to the TST. Indeterminate results were significantly associated with elderly and immunocompromised patients. Lymphocytopaenia and hypoalbuminaemia were significantly associated with indeterminate laboratory findings. When the QFT-2G test was repeated for all patients showing indeterminate results, $12(16.7 \%)$ patients demonstrated determinate results on the subsequent test.

Indeterminate results of the QuantiFERON TB-2G test under routine clinical practice are not infrequent. When scoring QuantiFERON TB-2G test results for elderly and immunocompromised patients, one must be careful because the possibility of obtaining determinate results may be low even if the test is repeated.

KEYWORDS: Elderly, immunocompromised, indeterminate result, QuantiFERON TB-2G, tuberculin skin test

ndeterminate results have been reported with a frequency of $5-40 \%$ for the QuantiFERON TB-2G (QFT-2G; Cellestis Ltd, Carnegie, Australia) test [1-5]. The present authors assessed the risk factors that can influence indeterminate results when using the QFT-2G test in routine clinical practice and compared the clinical characteristics of patients with indeterminate results from the QFT-2G test to those of patients with determinate results from the QFT-2G test.

\section{SUBJECTS AND METHODS}

The current study was approved by the Ethics Committee of the Kawasaki Medical School (Kurashiki, Japan). In total, 704 patients with suspected tuberculosis (TB) and latent $\mathrm{TB}$ infection (LTBI) were prospectively enrolled between January 2005 and December 2007. Subjects had been consulted at the Kawasaki Medical School Hospital, Kawasaki Medical School Kawasaki Hospital (Okayama, Japan), Kurashiki Central Hospital (Kurashiki, Japan),
Kurashiki Daiichi Hospital (Kurashiki, Japan) and Asahigaoka Hospital (Okayama, Japan). Informed written consent was obtained from all participants in the present study. All patients, except for five, had negative serological tests for HIV or showed an absence of obvious risk factors for HIV infection. Demographic, clinical, radiological and microbiological data were collected for all patients. Collected data included any history of a previous TB disease and risk factors for TB and LTBI. A diagnosis of TB was definitively confirmed by culture of sputum, bronchoalveolar lavage fluid or pleural fluid samples that were positive for Mycobacterium tuberculosis microbiologically.

\section{Tuberculin skin test}

For the tuberculin skin test (TST), $0.1 \mathrm{~mL}$ of tuberculin purified protein derivative (PPD; Nippon BCG, Tokyo, Japan; equivalent to three tuberculin units of PPD solution) was injected intradermally into the volar aspect of the forearm, and the transverse induration diameter was

\section{AFFILIATIONS}

Division of Respiratory Diseases, Dept of Medicine, Kawasaki Medical School, Matsushima, Kurashiki, Japan.

\section{CORRESPONDENCE}

Y. Kobashi

Division of Respiratory Diseases,

Dept of Medicine

Kawasaki Medical School

577 Matsushima

Kurashiki

Japan

Fax: 81864641041

E-mail: yoshihiro@

med.kawasaki-m.ac.jp

Received:

May 152008

Accepted after revision:

November 182008

STATEMENT OF INTEREST

None declared. 
measured $48 \mathrm{~h}$ later. The TST results were interpreted according to the level of risk, as reported in current guidelines [6].

\section{The QFT-2G test}

The QFT-2G test was performed according to the recommendations of the manufacturer. This judgment was performed according to the guidelines proposed by the Centers for Disease Control and Prevention (CDC) for using the QFT-2G test [7]. The QFT-2G test was repeated for all patients with indeterminate results, within 1 month.

\section{Statistical analysis}

Information from the questionnaires, TST results and wholeblood interferon (IFN)- $\gamma$ assay results were subjected to statistical analysis. The QFT-2G and TST results or indeterminate and determinate results of the QFT-2G test were compared using the Chi-squared test. The Wilson score method was used to calculate $95 \%$ confidence intervals for the positive rate of both tests [8].

\section{RESULTS}

The QFT-2G test and the TST results for patients suspected of $\mathrm{TB}$ and LTBI or confirmed to have TB are shown table 1. Among the 704 patients suspected of TB and LTBI, 72 patients were judged indeterminate with the QFT-2G test. The judgement of indeterminate result was attributed to positive control failure in 68 patients and negative control failure in the remaining four patients. Eight patients had positive TST results despite indeterminate results from the QFT-2G test. These eight patients showed a correlation with risk factors for TB and LTBI and radiological findings suggestive of previous TB disease; seven of these eight patients had previously received TB treatment. Among the 130 patients with confirmed TB disease, $14(10.8 \%)$ patients demonstrated indeterminate results from the QFT-2G test and they all showed a negative TST response. The rate of positive QFT-2G test results $(83.1 \%)$ was significantly higher compared with TST $(60.0 \%)$ results in 130 patients with confirmed TB disease. Although the QFT-2G test was repeated within 1 month for 72 patients showing indeterminate results, only $12(16.7 \%)$ patients showed determinate results on the subsequent test (two positive results and 10 negative results). Although two patients showed

\begin{tabular}{llcc}
\hline TABLE 1 & $\begin{array}{l}\text { QuantiFERON TB-2G (QFT-2G) test and } \\
\text { tuberculin skin test (TST) results for } 704 \text { patients } \\
\text { suspected of tuberculosis (TB) infection and } 130 \\
\text { patients with confirmed TB disease }\end{array}$ \\
\cline { 2 - 4 } QFT-2G & \multicolumn{3}{c}{ TST } \\
\cline { 2 - 4 } & Positive & Negative & Total \\
\hline Indeterminate & $8(0)$ & $64(14)$ & $72(14)$ \\
Determinate & $274(78)$ & $358(38)$ & $632(116)$ \\
Positive & $104(78)$ & $32(30)$ & $136(108)$ \\
Negative & $170(0)$ & $326(8)$ & $496(8)$
\end{tabular}

Data are presented as suspected TB results (confirmed TB results). QFT-2G was manufactured by Cellestis Ltd (Carnegie, Australia). positive results after the initial indeterminate results from the QFT-2G test at the first examination, the reason was suspected to be the discontinuation of immunosuppressive drugs for the underlying disease and recovery of the number of lymphocytes (from 210 to $860 \mu \mathrm{L}^{-1}$ and from 640 to $1,012 \mu \mathrm{L}^{-1}$ ).

The age distribution of patients showing indeterminate results on the QFT-2G test is shown in figure 1. Indeterminate results were significantly more frequent among elderly patients $(\geqslant 80$ yrs old) than among other age groups. Although indeterminate results in children $(<10$ yrs old) were more frequent than in other age groups, there was no significant difference.

There were no significant differences by year (2005, 2006 and 2007) or by season (January-March, April-June, JulySeptember and October-December) for transitional changes in the rate of indeterminate results on the QFT-2G test.

The clinical characteristics of 72 patients with indeterminate QFT-2G test results and the remaining 632 patients with determinate QFT-2G test results are shown in table 2. The mean age of patients showing indeterminate results was significantly higher than those showing determinate results $(p=0.012)$. Concerning underlying diseases, the number of patients receiving immunosuppressive treatment was significantly higher among patients showing indeterminate results than among those showing determinate results $(p=0.0008)$. However, the number of patients without underlying diseases was significantly higher among patients with determinate results than among those with indeterminate results $(\mathrm{p}=0.009)$.

The laboratory and radiological findings of 72 patients with indeterminate QFT-2G test results showed that leukocytopaenia $(p=0.024)$, lymphocytopaenia $(p=0.011)$ including CD4 lymphocytopaenia $(p=0.007)$, hypoproteinaemia $(p=0.028)$, and hypoalbuminaemia $(p=0.033)$ were significantly recognised in patients with indeterminate results more frequently than in those with determinate results (table 2).

\section{DISCUSSION}

In previous reports, it has been indicated that the sensitivity and specificity of the QFT-2G test were $59-89 \%$ and $70-98 \%$, respectively [9-11]. The majority of the QFT-2G test was classified into positive or negative reactions and only a small proportion of QFT-2G tests were judged indeterminate. In the present study, $72(10.2 \%)$ of 704 patients suspected of TB and LTBI demonstrated indeterminate QFT-2G test results according to CDC guidelines [7]. In a previous report, FERRARA et al. [1] noted that indeterminate QFT-2G test results appeared in $21 \%$ of tests performed in routine clinical microbiological laboratories due to positive control failure and that indeterminate results were significantly overrepresented among patients receiving immunosuppressive treatments. However, there was no speculation concerning the reason.

Through the current large scale study, the importance of the clinical characteristics, highly advanced age and underlying disease in patients receiving immunosuppressive treatment, were first demonstrated as risk factors that can promote indeterminate QFT-2G test results. Otherwise, laboratory findings of lymphocytopaenia, including CD4 lymphocytopaenia, showing an immunosuppressive state, or hypoproteinaemia, 


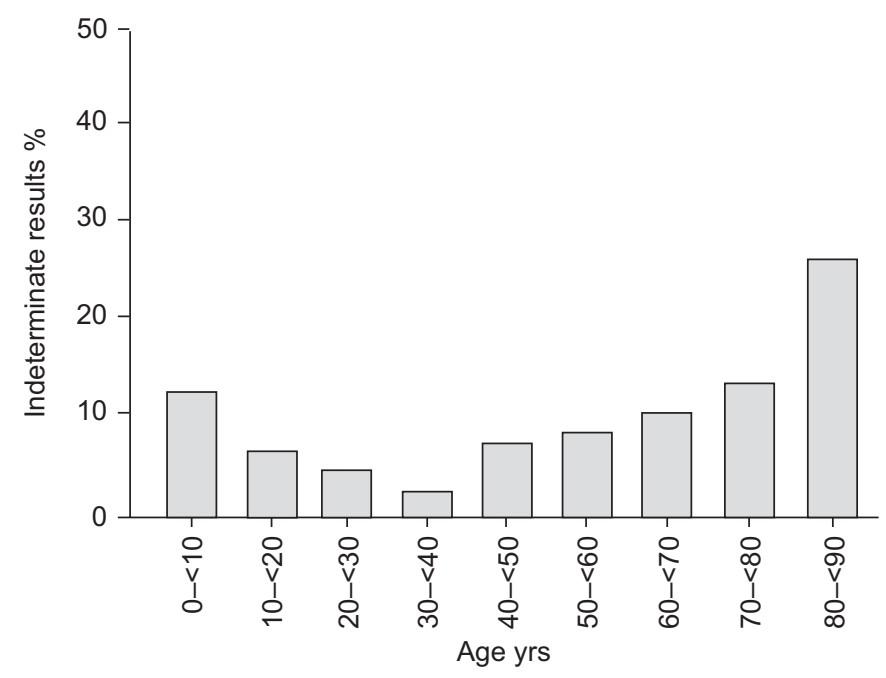

FIGURE 1. The proportion of indeterminate QuantiFERON TB-2G (Cellestis Ltd, Carnegie, Australia) test results in each age group.

showing a poor nutritional state, could significantly influence the risk of indeterminate results compared with determinate results. Immunosuppressive drugs, such as corticosteroid drugs or tumour necrosis factor (TNF)- $\alpha$ inhibitors, directly reduce the production of inflammatory cytokines, such as IFN- $\gamma$, interleukin-1 and TNF- $\alpha$ from T-lymphocytes similar to that in patients with lymphocytopaenia due to severe underlying diseases [7, 12, 13].

Sex, younger age, transitional changes during the four seasons over a 3-yr period, microbiological findings and radiological findings including extension of lesions do not appear to be associated with indeterminate results. In fact, all patients $>80$ yrs old had some form of severe underlying disease and most of these patients also had lymphocytopaenia or hypoproteinaemia as underlying diseases. However, although it has been indicated that younger children $(<5$ yrs old $)$ also tend to show indeterminate QFT-2G test results [14], this group presented with a normal range of lymphocyte counts and serum protein. There were no significant differences in the rate of these patients showing indeterminate results compared with the rates in other age groups. The QFT-2G test depends on the increased level of inflammatory cytokines secreted by T-lymphocytes previously sensitised to $M$. tuberculosis-specific antigens (early secretory antigenic target- 6 and culture filtrate protein-10). In the blood, mononuclear cells from peripheral blood are stimulated in vitro, and the production of IFN- $\gamma$ sensitised to M. tuberculosis-specific antigen is measured by ELISA in the QFT-2G test [15]. It was speculated that lymphocytopaenia caused a decrease in the production of IFN- $\gamma$ and induced indeterminate QFT-2G test results due to a lower mitogen level. For these reasons, indeterminate results due to positive control failure may be less frequent with the T-Spot.TB test (Oxford Immunotec Ltd, Abingdon, UK), which detects individual T-lymphocyte producing IFN- $\gamma$ by the ELISPOT technology, compared with that from the QFT-2G test $[3,16,17]$.

BEFFA et al. [18] reported that the rate of indeterminate results could be decreased to $3.4 \%$ by the T-Spot.TB test using the ELISPOT method. However, because the study by BEFFA et al. [18]

\begin{tabular}{|c|c|c|c|c|}
\hline \multicolumn{5}{|c|}{$\begin{array}{ll}\text { TABLE } 2 & \text { Clinical characteristics of } 72 \text { patients with } \\
\text { indeterminate QuantiFERON TB-2G (QFT-2G) } \\
\text { test results and the remaining } 632 \text { patients with } \\
\text { determinate QFT-2G test results }\end{array}$} \\
\hline \multirow{2}{*}{\multicolumn{2}{|c|}{ Clinical characteristics }} & \multicolumn{2}{|c|}{ QFT-2G test } & \multirow[t]{2}{*}{ p-value } \\
\hline & & Indeterminate & Determinate & \\
\hline Subjects $n$ & & 72 & 632 & \\
\hline Age yrs & & $68.2 \pm 11.0$ & $57.7 \pm 10.2$ & 0.013 \\
\hline Sex male/fer & & $42 / 30$ & $421 / 211$ & 0.105 \\
\hline Underlying o & sease & $68(94.4)$ & $494(78.2)$ & 0.081 \\
\hline Respiratory & isease & $7(9.7)$ & $107(16.1)$ & 0.094 \\
\hline Nonrespirat & ry disease & $61(84.7)$ & 387 (61.2) & 0.036 \\
\hline Malignan & disease $^{\#}$ & $13(18.1)$ & $102(16.1)$ & 0.843 \\
\hline Immunos & ppressive treatment & $30(41.7)$ & $71(11.2)$ & 0.0008 \\
\hline Diabetes & nellitus & $4(5.5)$ & $74(11.7)$ & 0.077 \\
\hline Chronic r & nal failure & $5(6.9)$ & $58(9.2)$ & 0.433 \\
\hline None & & $4(5.6)$ & $138(21.8)$ & 0.009 \\
\hline Received TB & treatment & $7(9.7)$ & $58(9.2)$ & 0.955 \\
\hline TB disease $^{+}$ & & $14(19.4)$ & $116(18.4)$ & 0.910 \\
\hline \multicolumn{5}{|c|}{ Laboratory findings } \\
\hline WBC count & cells $\cdot \mu \mathrm{L}^{-1}$ & $3880 \pm 506$ & $4554 \pm 592$ & 0.025 \\
\hline Lymphocyte & count cells $\cdot \mu \mathrm{L}^{-1}$ & $880 \pm 50$ & $1155 \pm 69$ & 0.011 \\
\hline CD4 lymph & cyte count cells $\cdot \mu \mathrm{L}^{-1}$ & $223 \pm 24$ & $402 \pm 28$ & 0.007 \\
\hline Total protei & $\mathrm{g} \cdot \mathrm{dL}^{-1}$ & $6.0 \pm 1.5$ & $6.7 \pm 1.6$ & 0.028 \\
\hline Albumin $\mathrm{g} \cdot \mathrm{C}$ & & $3.2 \pm 0.8$ & $3.7 \pm 0.8$ & 0.033 \\
\hline \multicolumn{5}{|c|}{ Microbiological findings ${ }^{\S}$} \\
\hline Smear posi & ve, culture positive & $7(50.0)$ & $61(52.6)$ & 0.891 \\
\hline Smear neg & ive, culture positive & $7(50.0)$ & $55(47.4)$ & 0.845 \\
\hline \multicolumn{5}{|c|}{ Radiological findings $f$} \\
\hline \multicolumn{5}{|c|}{ Extension of lesion $\# \#$} \\
\hline 1 & & $19(47.5)$ & $179(45.5)$ & 0.923 \\
\hline 2 & & $18(45.0)$ & $193(49.1)$ & 0.882 \\
\hline 3 & & $3(7.5)$ & $21(5.3)$ & 0.641 \\
\hline
\end{tabular}

Data are presented as mean \pm SD or $n(\%)$, unless otherwise stated. TB: tuberculosis; WBC: white blood cells. ${ }^{\#}$ : patients with a diagnosis of malignant disease who were receiving anti-cancer therapy $(n=30)$ and those who were not receiving anti-cancer therapy $(n=85)$. ? patients receiving systematic steroids $(n=70)$ or anti-tumour necrosis factor- $\alpha$ agents $(n=16)$, or steroids plus other immunosuppressive drugs $(n=15){ }^{+}$: Mycobacterium tuberculosis was identified from the culture of clinical specimens. ${ }^{\S}$ : performed for 130 patients identified as having M. tuberculosis from the culture of clinical specimens. ${ }^{f}$ : performed for 433 patients clinically diagnosed as pulmonary tuberculosis. \#\#: 1: extension within one-third of the unilateral lung field; 2: within the unilateral lung field; and 3: beyond the unilateral lung field. QFT-2G was manufactured by Cellestis Ltd (Carnegie, Australia).

was retrospective, data on the immune status (CD4 lymphocyte count or TST) were not available for patients with indeterminate results. LIEBESCHUETZ et al. [19] previously reported results of the ELISPOT method for children with various severe underlying diseases other than HIV infection in a community hospital-based population with suspected TB and LTBI using the T-Spot.TB test instead of the QFT-2G test. The test used in the current study differs from the recent version of QuantiFERON-TB Gold In-Tube (QFT-3G; Cellestis, Carnagie, Australia) test. Because the QFT-3G test contains 
additional M. tuberculosis-specific antigen (TB7.7), it is speculated that the sensitivity is higher. Indeed, HARADA et al. [20] reported that the sensitivity of QFT-3G test was significantly higher than that of QFT-2G test (92.6 and $81.4 \%$, respectively), while the specificity of both tests was the same (98.8\%). A large scale study using the QFT-3G test should be performed to confirm a decreased frequency of indeterminate results on tests for TB and LTBI.

In conclusion, indeterminate QuantiFERON TB-2G test results in routine clinical practice are not infrequent. When scoring QuantiFERON TB-2G test results for the elderly and immunocompromised patients, one must be careful because the possibility of determinate QuantiFERON TB-2G test results may be low even if this test is repeated.

\section{ACKNOWLEDGEMENTS}

The present authors would like to thank T. Matsushima (Kurashiki Daiichi Hospital, Kurashiki, and Asahigaoka Hospital, Okayama, Japan), N. Okimoto (Kawasaki Medical School Kawasaki Hospital, Okayama, Japan) and T. Kageoka (Kurashiki Central Hospital, Kurashiki, Japan) for helpful comments.

\section{REFERENCES}

1 Ferrara G, Losi M, Meacci M, et al. Routine hospital use of a new commercial whole blood interferon- $\gamma$-assay for the diagnosis of tuberculosis infection. Am J Respir Crit Care Med 2005; 172: 631-635.

2 Dogra S, Narang P, Mendiratta DK, et al. Comparison of a whole blood interferon $-\gamma$ assay with tuberculin skin testing for the detection of tuberculosis infection in hospitalized children in rural India. J Infect 2007; 54: 267-276.

3 Ferrara G, Losi M, D'Amico R, et al. Use in routine practice of two commercial blood tests for diagnosis of infection with Mycobacterium tuberculosis: a prospective study. Lancet 2006; 367: 1328-1334.

4 Connell TG, Curtis N, Ranganathan SC, Buttery JP. Performance of a whole blood interferon- $\gamma$ assay for detecting latent infection with Mycobacterium tuberculosis in children. Thorax 2006; 61: 616-620.

5 Mahomed H, Hughes EJ, Hawkridge T, et al. Comparison of mantoux skin test with three generations of a whole blood IFN- $\gamma$ assay for tuberculosis infection. Int $J$ Tuberc Lung Dis 2006; 10: 310-316.

6 American Thoracic Society. Targeted tuberculin testing and treatment of latent tuberculosis infection. Am J Respir Crit Care Med 2000; 161: S221-S247.

7 Mazurek GH, Jereb J, Lobue P, Iademarco MF, Metchock B, Vernon A. Division of Tuberculosis Elimination, National
Center for HIV, STD, and TB Prevention. Guidelines for using the QuantiFERON-TB Gold test for detecting Mycobacterium tuberculosis infection, United States. MMWR Recomm Rep 2005; 54: 49-55.

8 Newcombe RG. Two-sided confidence intervals for the single proportion: comparison of seven methods. Stat Med 1998; 17: 857-872.

9 Dewan PK, Grinsdale J, Kawamura LM. Low sensitivity of a whole-blood-interferon- $\gamma$ release assay for detection of active tuberculosis. Clin Infect Dis 2007; 44: 69-73.

10 Pai M, Riley LW, Colford JM Jr. Interferon- $\gamma$ assays in the immunodiagnosis of tuberculosis: a systematic review. Lancet Infect Dis 2004; 4: 761-776.

11 Pai M, Gokhale K, Joshi R, et al. Mycobacterium tuberculosis infection in health care workers in rural India: comparison of a whole-blood, interferon- $\gamma$ assay with tuberculin skin testing. JAMA 2005; 293: 2746-2755.

12 Guyre PM, Girard MT, Morganelli PM, Manganiello PD. Glucocorticoid effects on the production and actions of immune cytokines. J Steroid Biochem 1988; 30: 89-93.

13 Brack A, Rittner HL, Younge BR, Kaltschmidt C, Weyand $\mathrm{CM}$, Goronzy JJ. Glucocorticoid-mediated repression of cytokine gene transcription in human arteritis-SCID chimeras. J Clin Invest 2007; 99: 2842-2850.

14 Lalvani A, Millington KA. T-cell based diagnosis of childhood tuberculosis infection. Curr Opin Infect Dis 2007; 20: 264-271.

15 Andersen P, Munk ME, Pollock JM, Doherty TM. Specific immune-based diagnosis of tuberculosis. Lancet 2000; 256 1099-1104.

16 Lee JY, Choi HJ, Park IN, et al. Comparison of two commercial interferon- $\gamma$ assays for diagnosing Mycobacterium tuberculosis infection. Eur Respir J 2006; 28: 24-30.

17 Meier T, Eulenbruch HP, Wrighton-Smith P, Enders G, Regnath T. Sensitivity of a new commercial enzyme-linked immunospot assay (T SPOT-TB) for diagnosis of tuberculosis in clinical practice. Eur J Clin Microbiol Infect Dis 2005; 24: 529-536

18 Beffa $\mathrm{P}$, Zellweger A, Janssens JP, Wrighton-Smith $\mathrm{P}$, Zellweger JP. Indeterminate test results of T-SPOT.TB performed under routine field conditions. Eur Respir J 2008; 31: 842-846.

19 Liebeschuetz S, Bamber S, Ewer K, Deeks J, Pathan AA, Lalvani A. Diagnosis of tuberculosis in South African children with a T cell-based assay: a prospective cohort study. Lancet 2004; 364: 2196-2203.

20 Harada N, Higuchi K, Yoshiyama T, et al. Comparison of the sensitivity and specificity of two whole blood interferon- $\gamma$ assays for $M$. tuberculosis infection. I Infect 2008; 56: 348-353. 